\title{
Introduction to Stochastic Finance: Random Variables and Arbitrage Theory
}

\author{
Peter Jaeger \\ Siegmund-Schacky-Str. 18a \\ 80993 Munich, Germany
}

Summary. Using the Mizar system [1], 5], we start to show, that the Call-Option, the Put-Option and the Straddle (more generally defined as in the literature) are random variables (4, p. 15), see (Def. 1) and (Def. 2). Next we construct and prove the simple random variables (2], p. 14) in (Def. 8 ).

In the third section, we introduce the definition of arbitrage opportunity, see (Def. 12). Next we show, that this definition can be characterized in a different way (Lemma 1.3. in [4, p. 5), see (17). In our formalization for Lemma 1.3 we make the assumption that $\varphi$ is a sequence of real numbers (there are only finitely many valued of interest, the values of $\varphi$ in $R^{d}$ ). For the definition of almost sure with probability 1 see p. 6 in [2. Last we introduce the risk-neutral probability (Definition 1.4, p. 6 in [4), here see (Def. 16).

We give an example in real world: Suppose you have some assets like bonds (riskless assets). Then we can fix our price for these bonds with $x$ for today and $x \cdot(1+r)$ for tomorrow, $r$ is the interest rate. So we simply assume, that in every possible market evolution of tomorrow we have a determinated value. Then every probability measure of $\Omega_{f u t 1}$ is a risk-neutral measure, see (21). This example shows the existence of some risk-neutral measure. If you find more than one of them, you can determine - with an additional conidition to the probability measures - whether a market model is arbitrage free or not (see Theorem 1.6. in 4, p. 6.)

A short graph for (21):

Suppose we have a portfolio with many (in this example infinitely many) assets. For asset $d$ we have the price $\pi(d)$ for today, and the price $\pi(d) \cdot(1+r)$ for tomorrow with some interest rate $r>0$.

Let $\mathrm{G}$ be a sequence of random variables on $\Omega_{f u t 1}$, Borel sets. So you have many functions $f_{k}:\{1,2,3,4\} \rightarrow R$ with $G(k)=f_{k}$ and $f_{k}$ is a random variable of $\Omega_{f u t 1}$, Borel sets. For every $f_{k}$ we have $f_{k}(w)=\pi(k) \cdot(1+r)$ for $w \in\{1,2,3,4\}$. 


$$
\begin{aligned}
& \text { Today } \\
& \text { Tomorrow } \\
& \text { only one scenario } \quad\left\{\begin{array}{l}
w_{21}=\{1,2\}, \\
w_{22}=\{3,4\},
\end{array}\right. \\
& \text { for all } d \in \mathbb{N} \text { holds } \pi(d) \quad\left\{\begin{array}{l}
f_{d}(w)=G(d)(w)=\pi(d) \cdot(1+r), \\
w \in w_{21} \text { or } w \in w_{22}, \\
r>0 \text { is the interest rate. }
\end{array}\right.
\end{aligned}
$$

Here, every probability measure of $\Omega_{f u t 1}$ is a risk-neutral measure.

MSC: 28A05 03B35

Keywords: random variable; arbitrage theory; risk-neutral measure

MML identifier: FINANCE6, version: 8.1.07 5.47.1318

\section{Put-Option, Call-Option and Straddle are Random Variables}

From now on $\Omega$ denotes a non empty set and $F$ denotes a $\sigma$-field of subsets of $\Omega$.

Now we state the propositions:

(1) $] 0,+\infty[$ is an element of the Borel sets.

(2) Let us consider a random variable $R$ of $F$ and the Borel sets, an element $K$ of $\mathbb{R}$, and a function $g$ from $\Omega$ into $\mathbb{R}$. Suppose $g=$ $\chi_{(R-(\Omega \longmapsto K))^{-1}([0,+\infty[), \Omega}$. Then Call-Option $(R, K)=g \cdot(R-(\Omega \longmapsto K))$.

(3) Let us consider a random variable $R$ of $F$ and the Borel sets, and a real number $K$. Then $(\Omega \longmapsto K)-R$ is a random variable of $F$ and the Borel sets.

(4) Let us consider an element $A$ of $F$. Then $\chi_{A, \Omega}$ is a random variable of $F$ and the Borel sets.

(5) $\chi_{\Omega, \Omega}$ is random variable on $F$ and the Borel sets. The theorem is a consequence of (4).

(6) Let us consider random variables $f, R$ of $F$ and the Borel sets, and a real number $K$. Then $(f-R)^{-1}([0,+\infty[)$ is an element of $F$. The theorem is a consequence of (1).

Let us consider $\Omega$ and $F$. Let $R$ be a random variable of $F$ and the Borel sets and $K$ be a real number. Let us note that the functor Call-Option $(R, K)$ yields a random variable of $F$ and the Borel sets. The functor Put-Option $(R, K)$ yielding a function from $\Omega$ into $\mathbb{R}$ is defined by

(Def. 1) for every element $w$ of $\Omega$, if $((\Omega \longmapsto K)-R)(w) \geqslant 0$, then it $(w)=$ $((\Omega \longmapsto K)-R)(w)$ and if $((\Omega \longmapsto K)-R)(w)<0$, then it $(w)=0$. 
Now we state the proposition:

(7) Let us consider a random variable $R$ of $F$ and the Borel sets, a real number $K$, and a function $g$ from $\Omega$ into $\mathbb{R}$. Suppose $g=$ $\chi_{((\Omega \longmapsto K)-R)^{-1}([0,+\infty[), \Omega}$. Then Put-Option $(R, K)=g \cdot((\Omega \longmapsto K)-R)$.

Let us consider $\Omega$ and $F$. Let $R$ be a random variable of $F$ and the Borel sets and $K$ be a real number. Note that the functor Put-Option $(R, K)$ yields a random variable of $F$ and the Borel sets.

\section{Simple Random Variables}

Let us consider $\Omega$ and $F$. Let $R$ be a random variable of $F$ and the Borel sets and $K$ be a real number. The functor $\operatorname{Straddle}(R, K)$ yielding a random variable of $F$ and the Borel sets is defined by the term

(Def. 2) Put-Option $(R, K)+$ Call-Option $(R, K)$.

Now we state the proposition:

(8) Let us consider a random variable $R$ of $F$ and the Borel sets, a real number $K$, and an element $w$ of $\Omega$. Then $(\operatorname{Straddle}(R, K))(w)=\mid(R-$ $(\Omega \longmapsto K))(w) \mid$.

Let us consider $\Omega$ and $F$. The functors: the set of constants $F$ and the set of $\chi_{F}$ yielding sets are defined by terms

(Def. 3) $\{f$, where $f$ is a function from $\Omega$ into $\mathbb{R}: f$ is random variable on $F$ and the Borel sets and constant\},

(Def. 4) $\left\{\chi_{A, \Omega}\right.$, where $A$ is an element of $F: \chi_{A, \Omega}$ is random variable on $F$ and the Borel sets\},

respectively. Let $X$ be a set. We say that $X$ is $F$-random membered if and only if

(Def. 5) for every object $x$ such that $x \in X$ there exists a function $f$ from $\Omega$ into $\mathbb{R}$ such that $f=x$ and $f$ is random variable on $F$ and the Borel sets.

Observe that the set of constants $F$ is non empty and the set of $\chi_{F}$ is non empty and the set of constants $F$ is $F$-random membered and the set of $\chi_{F}$ is $F$-random membered and there exists a set which is $F$-random membered and non empty.

Let $D$ be an $F$-random membered, non empty set, $C_{1}$ be a sequence of $D$, and $n$ be a natural number. The change type of $C_{1}$ and $n$ yielding a random variable of $F$ and the Borel sets is defined by the term

(Def. 6) $C_{1}(n)$.

Let $C_{2}$ be a sequence of $D$ and $w$ be an element of $\Omega$. The change all types of $C_{2}$ and $w$ yielding a function from $\mathbb{N}$ into $\mathbb{R}$ is defined by 
(Def. 7) for every natural number $n$, it $(n)=\left(\right.$ the change type of $C_{2}$ and $\left.n\right)(w)$.

Let $D_{1}, D_{2}$ be $F$-random membered, non empty sets, $C_{1}$ be a sequence of $D_{1}, C_{2}$ be a sequence of $D_{2}$, and $n$ be a natural number. The simple $\mathcal{R} \mathcal{V}$ of $C_{1}$, $C_{2}$ and $n$ yielding a function from $\Omega$ into $\mathbb{R}$ is defined by

(Def. 8) for every element $w$ of $\Omega$,it $(w)=\left(\sum_{\alpha=0}^{\kappa}\left(\left(\right.\right.\right.$ the change all types of $C_{2}$ and $w) \cdot\left(\right.$ the change all types of $C_{1}$ and $\left.\left.\left.w\right)\right)(\alpha)\right)_{\kappa \in \mathbb{N}}(n)$.

Observe that the simple $\mathcal{R} \mathcal{V}$ of $C_{1}, C_{2}$ and $n$ yields a random variable of $F$ and the Borel sets.

\section{Arbitrage Theory: Definition and Alternative Representation}

From now on $\varphi$ denotes a sequence of real numbers and $\pi$ denotes a price function.

Let us consider $\Omega$ and $F$. Let $q$ be a natural number and $G$ be a sequence of the set of random variables on $F$ and the Borel sets. The change element to functions $G$ and $q$ yielding a real-valued random variable on $F$ is defined by the term

(Def. 9) $G(q)$.

Let us consider $\varphi$. Let $n$ be a natural number. The functors: the first $\mathcal{A O}$ set of $\varphi, \Omega, F, G$ and $n$ and the second $\mathcal{A O}$-set of $\varphi, \Omega, F, G$ and $n$ yielding elements of $F$ are defined by terms

(Def. 10) (the $\mathcal{R} \mathcal{V}$-portfolio value for future extension of $\varphi, F, G$ and $n)^{-1}([0,+\infty[$ ),

(Def. 11) (the $\mathcal{R} \mathcal{V}$-portfolio value for future extension of $\varphi, F, G$ and $n)^{-1}(] 0,+\infty[$ ), respectively. Let $P$ be a probability on $F$ and $\pi$ be a price function. We say that there exists an $\mathcal{A O}$ w.r.t. $P, G, \pi$ and $n$ if and only if

(Def. 12) there exists a sequence $\varphi$ of real numbers such that the buy portfolio extension of $\varphi, \pi$, and $n \leqslant 0$ and $P$ (the first $\mathcal{A O}$-set of $\varphi, \Omega, F, G$ and $n)=1$ and $P($ the second $\mathcal{A O}$-set of $\varphi, \Omega, F, G$ and $n)>0$.

Let $r$ be a real number. The first $\mathcal{R} \mathcal{V}$ of $r$ yielding an element of the set of random variables on $\Omega_{\text {now }}$ and the Borel sets is defined by the term

(Def. 13) $\{1,2,3,4\} \longmapsto r$.

Let $\pi$ be a price function and $d$ be a natural number. The first $\mathcal{R} \mathcal{V}$ of $\pi, r$ and $d$ yielding an element of the set of random variables on $\Omega_{f u t 1}$ and the Borel sets is defined by the term

(Def. 14) the first $\mathcal{R} \mathcal{V}$ of $\pi(d) \cdot(1+r)$.

Now we state the propositions:

(9) There exists a sequence $G$ of the set of random variables on $\Omega_{\text {now }}$ and the Borel sets such that 
(i) $G(0)=\{1,2,3,4\} \longmapsto 1$, and

(ii) $G(1)=\{1,2,3,4\} \longmapsto 5$, and

(iii) for every natural number $k$ such that $k>1$ holds $G(k)=$ $\{1,2,3,4\} \longmapsto 0$.

Proof: Define $\mathcal{U}$ (natural number) $=\left(\$_{1}=0 \rightarrow\right.$ the first $\mathcal{R} \mathcal{V}$ of $1,\left(\$_{1}=\right.$ $1 \rightarrow$ the first $\mathcal{R} \mathcal{V}$ of 5 , the first $\mathcal{R} \mathcal{V}$ of 0$)$ ). Consider $f$ being a sequence of the set of random variables on $\Omega_{\text {now }}$ and the Borel sets such that for every element $d$ of $\mathbb{N}, f(d)=\mathcal{U}(d) . f(0)=(0=0 \rightarrow$ the first $\mathcal{R} \mathcal{V}$ of $1,(0=$ $1 \rightarrow$ the first $\mathcal{R} \mathcal{V}$ of 5 , the first $\mathcal{R} \mathcal{V}$ of 0$)) . f(1)=(1=0 \rightarrow$ the first $\mathcal{R} \mathcal{V}$ of $1,(1=1 \rightarrow$ the first $\mathcal{R} \mathcal{V}$ of 5 , the first $\mathcal{R} \mathcal{V}$ of 0$))$. For every natural number $k$ such that $k>1$ holds $f(k)=\{1,2,3,4\} \longmapsto 0$.

(10) Let us consider a probability $P$ on $\Omega_{\text {now }}$, and a sequence $G$ of the set of random variables on $\Omega_{\text {now }}$ and the Borel sets. Suppose $G(0)=\{1,2,3,4\} \longmapsto$ 1 and $G(1)=\{1,2,3,4\} \longmapsto 5$ and for every natural number $k$ such that $k>1$ holds $G(k)=\{1,2,3,4\} \longmapsto 0$. Then there exists a price function $\pi$ such that there exists an $\mathcal{A O}$ w.r.t. $P, G, \pi$ and 1 .

Proof: Set $\Omega=\{1,2,3,4\}$. Set $F=\Omega_{\text {now }} P(\Omega)=1$ and $P(\emptyset)=0$. Define $\mathcal{U}$ (element of $\mathbb{N})=\left(\$_{1}=0 \rightarrow 1,\left(\$_{1}=1 \rightarrow 1,0\right)\right)(\in \mathbb{R})$. Consider $f$ being a function from $\mathbb{N}$ into $\mathbb{R}$ such that for every element $d$ of $\mathbb{N}, f(d)=$ $\mathcal{U}(d) . f$ is a price function. Reconsider $\pi=f$ as a price function. Define $\mathcal{U}($ element of $\mathbb{N})=\left(\$_{1}=0 \rightarrow-1,\left(\$_{1}=1 \rightarrow 1,0\right)\right)(\in \mathbb{R})$. Consider $\varphi$ being a sequence of real numbers such that for every element $k$ of $\mathbb{N}, \varphi(k)=\mathcal{U}(k)$. $P($ the first $\mathcal{A O}$-set of $\varphi, \Omega, F, G$ and 1$)=1$ and $P($ the second $\mathcal{A O}$-set of $\varphi, \Omega, F, G$ and 1$)>0$ and the buy portfolio extension of $\varphi, \pi$, and $1 \leqslant 0$ by [7, (9)].

(11) Let us consider a natural number $n$, a real number $r$, and a sequence $G$ of the set of random variables on $F$ and the Borel sets. Then $\{w$, where $w$ is an element of $\Omega$ : the portfolio value for future extension of $n, \varphi, F$, $G$ and $w \geqslant 0\}=($ the $\mathcal{R} \mathcal{V}$-portfolio value for future extension of $\varphi, F, G$ and $n)^{-1}([0,+\infty[)$. The theorem is a consequence of $(1)$.

Let us consider natural numbers $d, d_{1}$, a real number $r$, and a sequence $G$ of the set of random variables on $F$ and the Borel sets.

(12) Suppose $d_{1}=d-1$. Then $\{w$, where $w$ is an element of $\Omega$ : the portfolio value for future of $d, \varphi, F, G$ and $w \geqslant(1+r) \cdot$ (the buy portfolio of $\varphi, \pi$, and $d)\}=(($ the $\mathcal{R} \mathcal{V}$-portfolio value for future of $\varphi, F, G$ and $\left.d_{1}\right)-(\Omega \longmapsto(1+r) \cdot($ the buy portfolio of $\varphi, \pi$, and $\left.d))\right)^{-1}([0,+\infty[)$.

Proof: Set $S_{1}=\{w$, where $w$ is an element of $\Omega$ : the portfolio value for future of $d, \varphi, F, G$ and $w \geqslant(1+r)$. (the buy portfolio of $\varphi, \pi$, and $d)\}$. Set $S_{2}=(($ the $\mathcal{R} \mathcal{V}$-portfolio value for future of $\varphi, F, G$ and 
$\left.d_{1}\right)-(\Omega \longmapsto(1+r) \cdot($ the buy portfolio of $\varphi, \pi$, and $\left.d))\right)^{-1}([0,+\infty[)$. For every object $x, x \in S_{1}$ iff $x \in S_{2}$.

(13) ((The $\mathcal{R} \mathcal{V}$-portfolio value for future of $\varphi, F, G$ and $\left.d_{1}\right)-(\Omega \longmapsto(1+$ $r) \cdot($ the buy portfolio of $\varphi, \pi$, and $d)))^{-1}([0,+\infty[)$ is an event of $F$.

(14) Let us consider a natural number $d$, a real number $r$, and a sequence $G$ of the set of random variables on $F$ and the Borel sets. Then $\{w$, where $w$ is an element of $\Omega$ : the portfolio value for future extension of $d, \varphi, F$, $G$ and $w>0\}=($ the $\mathcal{R} \mathcal{V}$-portfolio value for future extension of $\varphi, F, G$ and $d)^{-1}(] 0,+\infty[)$. The theorem is a consequence of (1).

Let us consider natural numbers $d, d_{1}$, a real number $r$, and a sequence $G$ of the set of random variables on $F$ and the Borel sets.

(15) Suppose $d_{1}=d-1$. Then $\{w$, where $w$ is an element of $\Omega$ : the portfolio value for future of $d, \varphi, F, G$ and $w>(1+r) \cdot$ (the buy portfolio of $\varphi, \pi$, and $d)\}=(($ the $\mathcal{R} \mathcal{V}$-portfolio value for future of $\varphi, F, G$ and $\left.d_{1}\right)-(\Omega \longmapsto(1+r) \cdot($ the buy portfolio of $\varphi, \pi$, and $\left.d))\right)^{-1}(] 0,+\infty[)$.

Proof: Set $S_{1}=\{w$, where $w$ is an element of $\Omega$ : the portfolio value for future of $d, \varphi, F, G$ and $w>(1+r) \cdot($ the buy portfolio of $\varphi, \pi$, and $d)\}$. Set $S_{2}=(($ the $\mathcal{R} \mathcal{V}$-portfolio value for future of $\varphi, F, G$ and $\left.d_{1}\right)-(\Omega \longmapsto(1+r) \cdot($ the buy portfolio of $\varphi, \pi$, and $\left.d))\right)^{-1}(] 0,+\infty[)$. For every object $x, x \in S_{1}$ iff $x \in S_{2}$.

(16) ((The $\mathcal{R} \mathcal{V}$-portfolio value for future of $\varphi, F, G$ and $\left.d_{1}\right)-(\Omega \longmapsto(1+$ $r) \cdot($ the buy portfolio of $\varphi, \pi$, and $d)))^{-1}(] 0,+\infty[)$ is an event of $F$.

(17) Let us consider a price function $\pi$, and natural numbers $d, d_{1}$. Suppose $d>0$ and $d_{1}=d-1$. Let us consider a probability $P$ on $F$, and a real number $r$. Suppose $r>-1$. Let us consider a sequence $G$ of the set of random variables on $F$ and the Borel sets. Suppose the element of $F$, the Borel sets, $G$, and $0=\Omega \longmapsto 1+r$. Then there exists an $\mathcal{A O}$ w.r.t. $P, G, \pi$ and $d$ if and only if there exists a sequence $\varphi_{1}$ of real numbers such that $P\left(\left(\left(\right.\right.\right.$ the $\mathcal{R} \mathcal{V}$-portfolio value for future of $\varphi_{1}, F, G$ and $\left.d_{1}\right)-\left(\Omega \longmapsto(1+r) \cdot\left(\right.\right.$ the buy portfolio of $\varphi_{1}, \pi$, and $\left.\left.\left.d\right)\right)\right)^{-1}([0,+\infty[))=1$ and $P\left(\left(\left(\right.\right.\right.$ the $\mathcal{R} \mathcal{V}$-portfolio value for future of $\varphi_{1}, F, G$ and $\left.d_{1}\right)-(\Omega \longmapsto$ $(1+r) \cdot\left(\right.$ the buy portfolio of $\varphi_{1}, \pi$, and $\left.\left.\left.\left.d\right)\right)\right)^{-1}(] 0,+\infty[)\right)>0$.

Proof: If there exists an $\mathcal{A O}$ w.r.t. $P, G, \pi$ and $d$, then there exists a sequence $\varphi_{1}$ of real numbers such that $P(($ the $\mathcal{R} \mathcal{V}$-portfolio value for future of $\varphi_{1}, F, G$ and $\left.d_{1}\right)-(\Omega \longmapsto(1+r) \cdot$ (the buy portfolio of $\varphi_{1}, \pi$, and $\left.\left.\left.d\right)\right)\right)^{-1}([0,+\infty[))=1$ and $P((($ the $\mathcal{R} \mathcal{V}$-portfolio value for future of $\varphi_{1}, F, G$ and $\left.d_{1}\right)-\left(\Omega \longmapsto(1+r) \cdot\left(\right.\right.$ the buy portfolio of $\varphi_{1}$, $\pi$, and $\left.d)))^{-1}(] 0,+\infty[)\right)>0$. Define $\mathcal{U}$ (natural number) $=\left(\$_{1}=0 \rightarrow\right.$ -(the buy portfolio of $\varphi_{1}, \pi$, and $\left.\left.d\right), \varphi_{1}\left(\$_{1}\right)\right)(\in \mathbb{R})$. Consider $\varphi$ being a se- 
quence of real numbers such that for every element $n$ of $\mathbb{N}, \varphi(n)=\mathcal{U}(n)$. For every natural number $n$, if $n=0$, then $\varphi(n)=-$ (the buy portfolio of $\varphi_{1}, \pi$, and $d$ ) and if $n>0$, then $\varphi(n)=\varphi_{1}(n)$. The buy portfolio extension of $\varphi, \pi$, and $d=0$. $P$ (the first $\mathcal{A O}$-set of $\varphi, \Omega, F, G$ and $d)=1$. $P($ the second $\mathcal{A O}$-set of $\varphi, \Omega, F, G$ and $d)>0$.

\section{Risk-Neutral Probability Measure}

Let us consider $\Omega$ and $F$. Let $R$ be a real-valued random variable on $F$ and $r$ be a real number. The $r$-discounted value of $R$ yielding a real-valued random variable on $F$ is defined by the term

(Def. 15) $R \cdot \frac{1}{1+r}$.

Let $\pi$ be a price function and $G$ be a sequence of the set of random variables on $F$ and the Borel sets. We say that there exists a risk neutral measure w.r.t. $G, \pi$ and $r$ if and only if

(Def. 16) there exists a probability $P$ on $F$ such that for every natural number $d$, $\pi(d)=E_{P}$ the $r$-discounted value of (the change element to functions $G$ and $d)\}$.

From now on $P$ denotes a probability on $\Omega_{f u t 1}$.

Now we state the propositions:

(18) Let us consider a real number $r$. Suppose $r>0$. Let us consider a price function $\pi$, and a natural number $d$. Then there exists a real-valued random variable $f$ on $\Omega_{f u t 1}$ such that

(i) $f=\{1,2,3,4\} \longmapsto \pi(d) \cdot(1+r)$, and

(ii) $f$ is integrable on $\mathrm{P} 2 \mathrm{M}(P)$, and

(iii) $f$ is simple function in $\Omega_{f u t 1}$.

Proof: Set $\Omega_{2}=\{1,2,3,4\}$. Define $\mathcal{U}$ (element of $\left.\Omega_{2}\right)=\pi(d) \cdot(1+r)(\in \mathbb{R})$. Consider $f$ being a function from $\Omega_{2}$ into $\mathbb{R}$ such that for every element $d$ of $\Omega_{2}, f(d)=\mathcal{U}(d)$. Set $g=\Omega_{2} \longmapsto \pi(d) \cdot(1+r)(\in \mathbb{R})$. For every object $x$ such that $x \in \operatorname{dom} f$ holds $f(x)=g(x)$. $f$ is integrable on $\mathrm{P} 2 \mathrm{M}(P)$ by [6, (9), (3)], [3, (12)].

(19) Let us consider a natural number $n$, and a real number $r$. Suppose $r>0$. Let us consider a price function $\pi$, a natural number $d$, and a real-valued random variable $R$ on $\Omega_{f u t 1}$. Suppose $R=\{1,2,3,4\} \longmapsto \pi(d) \cdot(1+r)$ and $R$ is integrable on $\operatorname{P} 2 \mathrm{M}(P)$ and $R$ is simple function in $\Omega_{f u t 1}$. Then $\pi(d)=E_{P}\{$ the $r$-discounted value of $R\}$. 
Proof: Set $F=\Omega_{f u t 1} \cdot \overline{\mathbb{R}}(R)=R$ and $R$ is non-negative. Set $m=\pi(d)$. $(1+r)$. for every object $x$ such that $x \in \operatorname{dom} \overline{\mathbb{R}}(R)$ holds $(\overline{\mathbb{R}}(R))(x)=m$ and $\operatorname{dom} \overline{\mathbb{R}}(R) \in F$ and $0 \leqslant m$.

(20) Let us consider a real number $r$. Suppose $r>0$. Let us consider a price function $\pi$. Then there exists a sequence $G$ of the set of random variables on $\Omega_{f u t 1}$ and the Borel sets such that for every natural number $d, G(d)=$ $\{1,2,3,4\} \longmapsto \pi(d) \cdot(1+r)$ and the change element to functions $G$ and $d$ is integrable on $\mathrm{P} 2 \mathrm{M}(P)$ and the change element to functions $G$ and $d$ is simple function in $\Omega_{f u t 1}$.

Proof: Define $\mathcal{U}$ (natural number) $=$ the first $\mathcal{R} \mathcal{V}$ of $\pi, r$ and $\$_{1}$. Consider $g$ being a sequence of the set of random variables on $\Omega_{f u t 1}$ and the Borel sets such that for every element $d$ of $\mathbb{N}, g(d)=\mathcal{U}(d)$. There exists a realvalued random variable $R$ on $\Omega_{f u t 1}$ such that $R=\{1,2,3,4\} \longmapsto \pi(d)$. $(1+r)(\in \mathbb{R})$ and $R$ is integrable on $\operatorname{P} 2 \mathrm{M}(P)$ and $R$ is simple function in $\Omega_{\text {fut } 1}$.

(21) Let us consider a real number $r$. Suppose $r>0$. Let us consider a price function $\pi$, and a sequence $G$ of the set of random variables on $\Omega_{f u t 1}$ and the Borel sets. Suppose for every natural number $d, G(d)=\{1,2,3,4\} \longmapsto$ $\pi(d) \cdot(1+r)$ and the change element to functions $G$ and $d$ is integrable on $\mathrm{P} 2 \mathrm{M}(P)$ and the change element to functions $G$ and $d$ is simple function in $\Omega_{f u t 1}$. Then

(i) there exists a risk neutral measure w.r.t. $G, \pi$ and $r$, and

(ii) for every natural number $s, \pi(s)=E_{P}$ the $r$-discounted value of (the change element to functions $G$ and $s$ ) .

The theorem is a consequence of (19).

\section{REFERENCES}

[1] Grzegorz Bancerek, Czesław Byliński, Adam Grabowski, Artur Korniłowicz, Roman Matuszewski, Adam Naumowicz, Karol Pąk, and Josef Urban. Mizar: State-of-the-art and beyond. In Manfred Kerber, Jacques Carette, Cezary Kaliszyk, Florian Rabe, and Volker Sorge, editors, Intelligent Computer Mathematics, volume 9150 of Lecture Notes in Computer Science, pages 261-279. Springer International Publishing, 2015. ISBN 978-3319-20614-1. doi 10.1007/978-3-319-20615-8_17.

[2] Heinz Bauer. Wahrscheinlichkeitstheorie. de Gruyter-Verlag, Berlin, New York, 2002.

[3] Noboru Endou, Katsumi Wasaki, and Yasunari Shidama. The measurability of extended real valued functions Formalized Mathematics, 9(3):525-529, 2001.

[4] Hans Föllmer and Alexander Schied. Stochastic Finance: An Introduction in Discrete Time, volume 27 of Studies in Mathematics. de Gruyter, Berlin, 2nd edition, 2004.

[5] Adam Grabowski, Artur Korniłowicz, and Adam Naumowicz. Four decades of Mizar. Journal of Automated Reasoning, 55(3):191-198, 2015. doi 10.1007/s10817-015-9345-1

[6] Peter Jaeger. Elementary introduction to stochastic finance in discrete time. Formalized Mathematics, 20(1):1-5, 2012. doi 10.2478/v10037-012-0001-5. 
INTRODUCTION TO STOCHASTIC FINANCE: RANDOM VARIABLES AND $\ldots \quad 9$

[7] Peter Jaeger. Modelling real world using stochastic processes and filtration. Formalized Mathematics, 24(1):1-16, 2016. doi 10.1515/forma-2016-0001.

Received March 27, 2018 